\title{
Mobility edge of the two-dimensional Bose-Hubbard model
}

\author{
Andreas Geißler (1,2,* and Guido Pupillo ${ }^{1}$ \\ ${ }^{1}$ icFRC, ISIS, University of Strasbourg and CNRS, 67000 Strasbourg, France \\ ${ }^{2}$ Institut für Theoretische Physik, Goethe-Universität, 60438 Frankfurt/Main, Germany
}

(Received 8 December 2019; revised 29 September 2020; accepted 19 November 2020; published 8 December 2020)

\begin{abstract}
We analyze the disorder-driven localization of the two-dimensional Bose-Hubbard model by evaluating the full low-energy quasiparticle spectrum via a recently developed fluctuation operator expansion method. For any strength of the local interaction we find a mobility edge that displays an approximately exponential decay with increasing disorder strength. We determine the finite-size scaling collapse and exponents at this critical line finding that the localization of excitations is characterized by weak multifractality and a thermal-like critical gap ratio. A direct comparison to a recent experiment yields an excellent match of the predicted finite-size transition point and scaling of single particle correlations.
\end{abstract}

DOI: 10.1103/PhysRevResearch.2.042037

Introduction. In the last decade the study of disorder-driven localization of quantum particles has received considerable interest, following the suggestion that Anderson localization for noninteracting models [1-4] can be generalized to interacting ones [5-8] in the framework of the so-called many-body localization (MBL). One of the most prominent features of MBL is its incompatibility with the eigenstate thermalization hypothesis resulting from an extensive number of local integrals of motion [9-13]. A complete demonstration of MBL would in principle require knowledge of the whole spectrum, limiting the use of exact diagonalization techniques to small system sizes, especially when bosonic particles are considered [14,15]. The existence of MBL has been rigorously proven in one-dimensional (1D) spin chains [16,17], while various perturbative arguments $[3,5,6,18]$ and numerical evidence $[7,8,19]$ have also supported its existence in two dimensions - involving a mobility edge (ME) separating mobile from localized states in the spectrum. However, recent theoretical arguments have challenged the existence of MBL both in 1D [20] and 2D [21-23] in the thermodynamic limit. Experimental realizations of bosonic systems have already been achieved in cold atom setups where a disorder potential can be imprinted onto a confined optical lattice in 1D [24,25] and 2D [26,27], showing strong signs of high-energy localization in confined systems for both cases. Related experiments $[28,29]$ have also observed evidence for a ground state Boseglass (BG) phase compatible with theoretical predictions of a zero-energy superfluid to BG transition [30-39].

Here, we investigate localization effects in the excitation spectrum of the two-dimensional Bose-Hubbard model (BHM) in the presence of disorder utilizing a recently devel-

\footnotetext{
*andreas.geissler87@gmail.com
}

Published by the American Physical Society under the terms of the Creative Commons Attribution 4.0 International license. Further distribution of this work must maintain attribution to the author(s) and the published article's title, journal citation, and DOI. oped fluctuation operator expansion (FOE) method [40-43], which gives access to the complete spectrum of quasiparticle (QP) excitations for system sizes comparable to experiments. Our results are summarized in Fig. 1. For all interaction strengths disorder induces (at least) one ME. We determine the finite-size scaling at the critical points characterized by weak fractality and a thermal-like critical gap ratio. Importantly, in the limits of our numerical QP method the low-energy $\mathrm{ME}$ converges onto an exponential decay with disorder. For the case of particles confined by a harmonic potential, we compute correlation functions and extract the inverse decay length, finding excellent agreement with recent experiments [26] in terms of a finite-size localization transition.

In the following we first introduce the model (Sec. II) and the fluctuation operator expansion (Sec. III). In Sec. IV we introduce the observables used to characterize localization and discuss their finite-size collapse determining the ME (Sec. IV). We determine the finite-size localization transition of a harmonically trapped system (Sec. V) and directly compare to the experimental results of Ref. [26]. In Sec. VI finally, we end with some concluding remarks.

Model. The Hamiltonian of the BHM with on-site disorder and in the grand canonical ensemble reads

$$
\hat{H}=\sum_{\ell}^{L^{2}} \underbrace{\left(\mu_{\ell} \hat{b}_{\ell}^{\dagger} \hat{b}_{\ell}+\frac{U}{2} \hat{b}_{\ell}^{\dagger} \hat{b}_{\ell}^{\dagger} \hat{b}_{\ell} \hat{b}_{\ell}\right)}_{\hat{H}_{\ell}}-t \sum_{\left\langle\ell, \ell^{\prime}\right\rangle}\left(\hat{b}_{\ell}^{\dagger} \hat{b}_{\ell^{\prime}}+\text { H.c. }\right),
$$

where $\hat{b}_{\ell}^{\dagger}\left(\hat{b}_{\ell}\right)$ are bosonic creation (annihilation) operators at the site $\ell, t$ is the tunneling rate between nearest neighbor sites $\left\langle\ell, \ell^{\prime}\right\rangle$ on a square lattice of spacing $a$ and linear size $L$, while $U$ is the local on-site Hubbard interaction. The energy $\mu_{\ell}$ reads $\mu_{\ell}=-\mu+\epsilon_{\ell}$, with $\mu$ the chemical potential fixing the particle number and $\epsilon_{\ell}$ a local energy shift due to disorder or an external harmonic potential. With Ref. [26] in mind we choose a Gaussian probability distribution $P\left(\epsilon_{\ell}\right)=\left(2 \pi W^{2}\right)^{-1 / 2} \exp \left[-\epsilon_{\ell}^{2} /\left(2 W^{2}\right)\right]$ with the standard deviation $W$ [44]. In this work we analyze this model over a range of interactions $U / t \in[1,25]$ and disorder strengths 


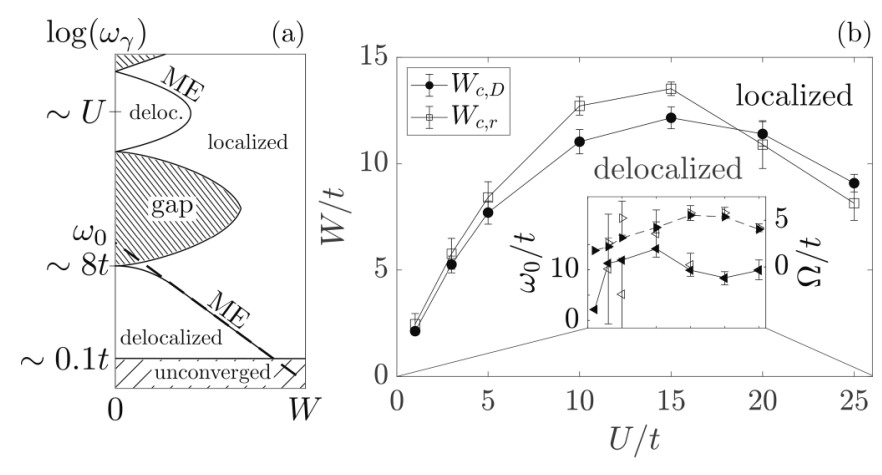

FIG. 1. Quasiparticle localization in the disordered 2D BoseHubbard model (1). (a) Typical structure of the quasiparticle spectrum at fixed interaction $U>8 t$. For sufficiently weak disorder $W$ a band gap persists, while multiple MEs centered at the homogeneous bands separate localized from delocalized states. The lowest ME displays exponential behavior (dashed line) down to the lowest resolved QP energies. (b) ME $W_{c}(\omega)$ of excitations at a quasiparticle energy of $\omega=t$ as a function of $U / t$, determined from the fractal dimension $D$ and the gap ratio $r$ (see legend). Inset: Amplitude $\omega_{0}$ (left-pointing triangles) and decay constant $\Omega$ (right-pointing triangles) characterizing the lowest ME (11).

$W / t \in[1,15]$ at half-filling. We furthermore investigate the effect of an external trapping potential in order to compare with the recent experiment [26] for $U=24.4 t$ and $W / t \in$ $[0.4,7]$.

Fluctuation operator expansion. The FOE [40-43] is a QP method based on a Gutzwiller expansion of (1) in terms of eigenstates $|i\rangle_{\ell}$ of the local mean-field Hamiltonians $\hat{H}_{\mathrm{MF}}^{(\ell)}=\hat{H}_{\ell}-t \sum_{\left\{\ell^{\prime} \mid\left\langle\ell, \ell^{\prime}\right\rangle\right\}}\left(\hat{b}_{\ell}^{\dagger} \phi_{\ell^{\prime}}+\right.$ H.c. $)$, where the fluctuation operators $\hat{\delta b_{\ell}} \equiv \hat{b}_{\ell}-\phi_{\ell}$ and the fields $\phi_{\ell} \stackrel{!}{=}$ ${ }_{\ell}\left\langle 0\left|\hat{b}_{\ell}\right| 0\right\rangle_{\ell}$ are determined self-consistently. For $N \rightarrow \infty$, $\hat{\delta b_{\ell}}=\sum_{i, j=0}^{N}\left\langle i\left|\hat{\delta b_{\ell}}\right| j\right\rangle_{\ell}|i\rangle_{\ell \ell}\langle j|$ constitutes an exact quadratic map onto a complete basis set of the local Gutzwiller raising (lowering) operators $\sigma_{\ell}^{(i)^{\dagger}} \equiv|i\rangle_{\ell \ell}\langle 0|\left(\sigma_{\ell}^{(i)} \equiv|0\rangle_{\ell \ell}\langle i|\right)$. These generate arbitrary local fluctuations $\kappa_{\ell}=\sum_{i>0} \sigma_{\ell}^{(i)^{\dagger}} \sigma_{\ell}^{(i)}$ of any self-consistent MF state $\left|\psi_{\mathrm{MF}}\right\rangle=\prod_{\ell}|0\rangle_{\ell}$. The quality of the approximation is ascertained for $\kappa=L^{-2} \sum_{\ell}\left\langle\kappa_{\ell}\right\rangle \ll 1$ [in this work we always find this criterion to be fulfilled in the quasiparticle ground state (5) [45]]. Here, we consider terms of second order in the Gutzwiller operators, which using $\sigma=\left(\sigma_{1}^{(1)}, \ldots, \sigma_{L^{2}}^{(N)}\right)^{\mathrm{T}}$ yields the following approximate representation of $\hat{H}[46]$ :

$$
\hat{\mathcal{H}}_{\mathrm{QP}}^{(2)} \equiv \frac{1}{2}\left(\begin{array}{c}
\sigma \\
\sigma^{\dagger}
\end{array}\right)^{\dagger}\left(\begin{array}{cc}
h & \Delta \\
\Delta^{*} & h^{*}
\end{array}\right)\left(\begin{array}{c}
\sigma \\
\sigma^{\dagger}
\end{array}\right)-\frac{1}{2} \operatorname{Tr}(h) .
$$

The scalar term $\operatorname{Tr}(h) / 2$ results from reordering normal ordered terms to antinormal order, while the matrix entries are given by the local matrix elements $B_{i, j}^{(\ell)} \equiv{ }_{\ell}\left\langle i\left|\hat{b}_{\ell}\right| j\right\rangle_{\ell}$,

$$
\begin{aligned}
h_{(i, \ell),\left(j, \ell^{\prime}\right)}= & -t_{\ell, \ell^{\prime}}\left(B_{0, i}^{(\ell) *} B_{0, j}^{\left(\ell^{\prime}\right)}+B_{i, 0}^{(\ell)} B_{j, 0}^{\left(\ell^{\prime}\right) *}\right) \\
& +\delta_{\ell, \ell^{\prime}} \delta_{i, j}\left(E_{i}^{(\ell)}-E_{0}^{(\ell)}\right), \\
\Delta_{(i, \ell),\left(j, \ell^{\prime}\right)}= & -t_{\ell, \ell^{\prime}}\left(B_{0, i}^{(\ell) *} B_{j, 0}^{\left(\ell^{\prime}\right)}+B_{i, 0}^{(\ell)} B_{0, j}^{\left(\ell^{\prime}\right) *}\right) .
\end{aligned}
$$

Here, $t_{\ell, \ell^{\prime}}$ is the tunneling matrix with nonzero entries only for neighboring sites, and $E_{i}^{(\ell)}$ are the local excitation energies of the $i$ th Gutzwiller excitation at site $\ell$.

The diagonalization of (2) yields $\hat{H} \approx \sum_{\gamma} \omega_{\gamma} \beta_{\gamma}^{\dagger} \beta_{\gamma}+$ $\Delta E_{\mathrm{QP}}$ in terms of infinitely lived QP modes $\gamma$ with corresponding energies $\omega_{\gamma}$ and $\beta_{\gamma} \equiv \mathbf{u}^{(\gamma)^{\dagger}} \boldsymbol{\sigma}+\mathbf{v}^{(\gamma)^{\dagger}} \boldsymbol{\sigma}^{\dagger}$ are the generalized Bogoliubov-type operators, with $\mathbf{u}^{(\gamma)}$ and $\mathbf{v}^{(\gamma)}$ the corresponding eigenvectors. Analogous to standard Bogoliubov theory, these inherit approximately bosonic commutation relations $\left[\beta_{\gamma}, \beta_{\gamma^{\prime}}^{\dagger}\right] \approx \delta_{\gamma, \gamma^{\prime}}$ from the Gutzwiller operators for $\left|\mathbf{u}^{(\gamma)}\right|^{2}-\left|\mathbf{v}^{(\gamma)}\right|^{2}=1$. $\mathbf{v}^{(\gamma)}$ and $\mathbf{u}^{(\gamma)}$ can be interpreted as dual wave functions analogous to particle and hole fluctuations. Normal ordering of operators results in a scalar correction $\Delta E_{\mathrm{QP}}$, irrelevant to the present discussion [41]. Finally, we implicitly define the QP ground state via

$$
\beta_{\gamma}\left|\psi_{\mathrm{QP}}\right\rangle=0 \quad \forall \gamma
$$

which also best fulfills the approximation of neglected QP interactions [40,41].

Drawing from variational concepts [47-50] and based on a MF description that becomes exact for weak and strong interactions, the FOE allows for a systematic, nonperturbative improvement over standard Bogoliubov theory [51] that also incorporates the effects of many-body entanglement [52-54]. It gives access to the otherwise neglected gapped (amplitude) Hubbard subbands in the disorder-free limit of $\hat{H}$ in Eq. (1) $[40,41]$. As we show in the following sections, these modes, absent in standard Bogolioubov theory, play an important role in the localization transition at finite energy. We note that Bogoliubov quasiparticle theory has already been used to successfully investigate 2D localization at low energy (e.g., $[37,38,55])$, and in particular the existence of a BG phase for hard-core bosons (i.e., $U \rightarrow \infty$ ) with binary disorder [37]. Numerous works have unambiguously demonstrated the existence of a direct zero-energy phase transition between a Bose condensed superfluid and a BG for the 2D BHM with uniform disorder distribution, similar to Eq. (1) [31-33,35,36,39]. Here we focus on the existence of a finite-energy ME.

Localization characteristics. To characterize the degree of localization we consider the following two observables: (i) The gap ratio

$$
r_{\gamma} \equiv\left\langle\frac{\min \left[\Delta \omega_{\gamma-1}, \Delta \omega_{\gamma}\right]}{\max \left[\Delta \omega_{\gamma-1}, \Delta \omega_{\gamma}\right]}\right\rangle_{d}
$$

with $\Delta \omega_{\gamma}=\omega_{\gamma+1}-\omega_{\gamma}$ the quasiparticle energy gaps and $\langle\cdot\rangle_{d}$ the disorder average. The observable $r_{\gamma}$ is known from random matrix theory [7,56] to have the mean value $r_{\mathrm{G}} \approx 0.5307$ and $r_{\mathrm{P}}=2 \ln 2-1 \approx 0.3863$ in the delocalized and localized phases, respectively, resulting from level statistics belonging to the Gaussian orthogonal and Poisson ensembles. The second observable is (ii) the fractal dimension $D_{q=2}^{(\gamma)}$ of the QP fluctuation wave functions $\mathbf{v}^{(\gamma)}$. Analogous to the scaling of $q$ moments $R_{q}=\sum_{n}\left|\psi_{n}\right|^{2 q}$ of many-body eigenstates [57-59] we define

$$
D_{q=2}^{(\gamma)}=\left\langle-\log _{L^{2}} \frac{\sum_{\ell}^{L^{2}}\left|\mathbf{v}_{\ell}^{(\gamma)}\right|^{4}}{\sum_{\ell}^{L^{2}}\left|\mathbf{v}_{\ell}^{(\gamma)}\right|^{2}}\right\rangle_{d},
$$



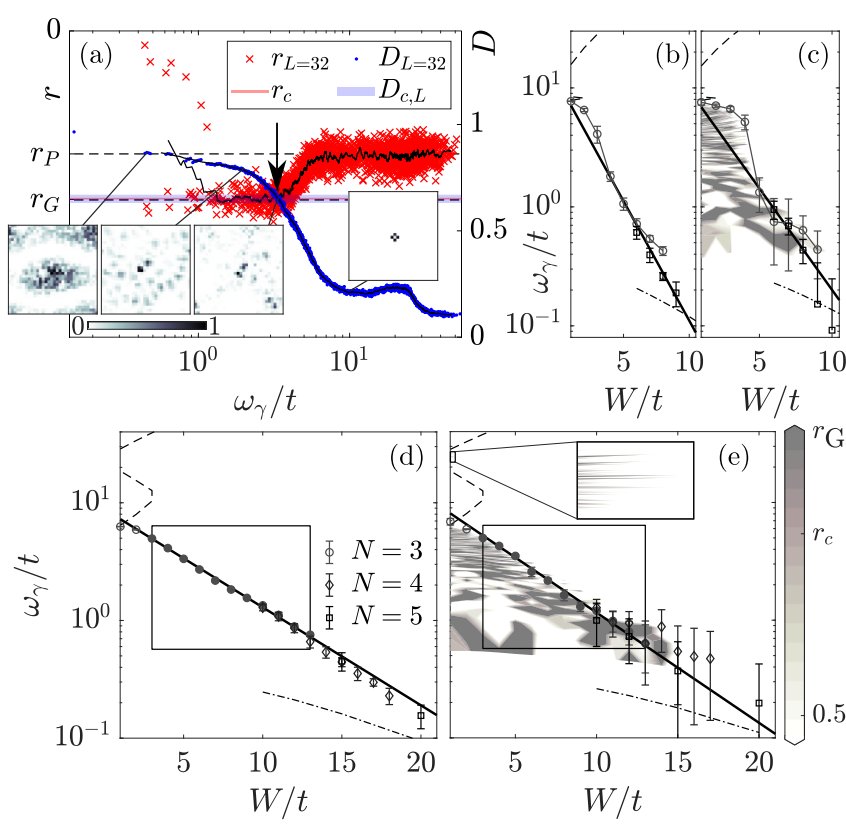

$W / t \quad W / t$

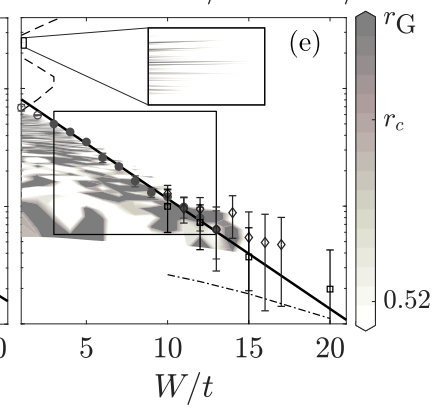

FIG. 2. (a) Gap ratio $r$ (left ordinate, inverted) and fractal dimension $D$ data (right ordinate) as functions of the QP energy $\omega_{\gamma} / t$ for $U / t=20, W / t=5$, and $L=32$ averaged over 95 realizations. Black lines are moving averages (of 21 points) as a guide to the eye and dashed lines mark $r_{\mathrm{P}}$ and $r_{\mathrm{G}}$. The crossing point (vertical arrow) of the data with the critical $r_{c}$ (shaded red, narrow) and $D_{\mathrm{c}, \mathrm{L}}$ (shaded blue, wide) mark the ME. Insets: Exemplary squared QP wave functions $\left|\mathbf{v}_{\ell}^{(\gamma)}\right|^{2}$ with maxima normalized to 1. (b)-(e) Separation of the QP spectra by the ME for $U / t=3[(\mathrm{~b})$, (c)] and $U / t=20[(\mathrm{~d}),(\mathrm{e})]$. Dashed lines mark band edges, dash-dotted lines signify lowest resolved energies for $N=5$ (dashed regions; see Supplemental Material [52]), while data points mark the ME with the respective FOE truncation given in the legend of (d). Panels (c) and (e) are contour plots of $r$ [color scale in (e)] binned under the condition $r>0.3$ (see text). Inset (e) shows remnants of a ME for the upper bands, while large boxes in (d) and (e) mark the region for which finite-size scaling has been performed, yielding the filled data points. Thick black lines in (b)-(e) are fits of Eq. (11) (see text).

for the local amplitudes $\left|\mathbf{v}_{\ell}^{(\gamma)}\right|^{2}=\sum_{i>0}\left|\mathbf{v}_{\ell, i}^{(\gamma)}\right|^{2}$ of the wave function, which naturally characterize the spatial extension of each QP mode [see examples in Fig. 2(a)]. For our purposes we consider $q=2$, while one obtains the multifractality spectrum by also taking all other values $q>0$ into account.

Delocalized states with $r \approx r_{\mathrm{G}}[60]$ appear primarily at low QP energies $\omega_{\gamma} / t$ and for weak disorder $W / t$, as shown in the contour plots Figs. 2(c) and 2(e) for weak $(U=3 t)$ and strong $(U=20 t)$ interactions, respectively. We note that values of $r<r_{\mathrm{P}}$ for weak disorder and small QP energies [e.g., for $\omega_{\gamma} \lesssim t$ in Fig. 2(a)] result from symmetry related finite-size effects irrelevant to our discussion.

For $U / t \gtrsim 20$ and $W / t \lesssim 1$ we find a band of additional delocalized states for energies $\omega_{\gamma} \sim U$ reflecting the presence of typical Hubbard subbands which overlap for $U \lesssim t$ [dashed lines in Figs. 2(b)-2(e)]. In all cases, increasing $W / t$ spreads the bands so they overlap and drives a transition to localized states with $r_{\mathrm{G}} \gtrsim r \geqslant r_{\mathrm{P}}$, implying the existence of (multiple) MEs. For the same cases we find similar behavior for the fractal dimension $D$ down to the truncation limit [compare
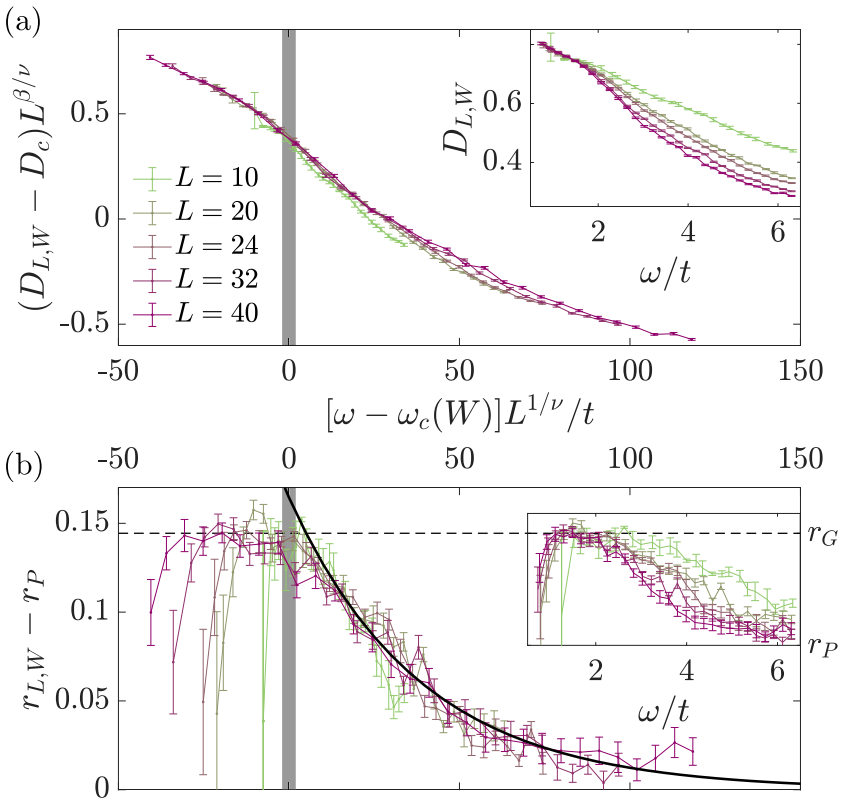

FIG. 3. Exemplary scaling collapse of $D$ (a) and $r$ (b). For the finite-size scaling every data set is binned for 30 equal spaced energies [within the region shown in Figs. 2(d) and 2(e)], while $L \in[10,20,24,32,40]$ [legend in (a)] with bins containing $[4,8,12,20,32]$ disorder averaged data values closest in $\omega_{\gamma}$ to $\omega$, respectively, and $W / t=7$. In (b) the horizontal dashed line marks $r_{\mathrm{G}}-r_{\mathrm{P}}$, the solid line is an exponential fit as a guide for the eye, and data within the gray shaded regions is used to determine $r_{c}$ and $\left\langle\tilde{D}_{W}(0)\right\rangle_{M E}$. Insets show unscaled data.

Figs. 2(b) and 2(d)]. Data points in Figs. 2(b)-2(d) mark the MEs determined via a finite-size scaling as discussed in the next section.

Finite-size scaling analysis. We determine the position of the (lowest energy) ME via finite-size scaling for the case $U=$ $20 t$, with linear sizes $L \in\{10,20,24,32,40\}$ and corresponding numbers of realizations $N_{r} \in\{480,240,240,95,48\}$ for $N=3$ which we find to be sufficient here [52]. We find the data to be consistent with the scaling relations

$$
\begin{gathered}
r_{L, W}(\omega)=\tilde{r}_{W}\left(\left[\omega-\omega_{c}(W)\right] L^{1 / v}\right), \\
D_{L, W}(\omega)-D_{c}=L^{-\beta / v} \tilde{D}_{W}\left(\left[\omega-\omega_{c}(W)\right] L^{1 / \nu}\right) .
\end{gathered}
$$

Here, $\tilde{r}_{W}(\cdot)$ and $\tilde{D}_{W}(\cdot)$ are the scaling functions, while the universal scaling exponents $\{\beta, \nu\}$ and the critical fractal dimension $D_{c}$ are to be determined self-consistently in combination with the critical energies $\omega_{c}(W)$ corresponding to the ME. Figure 3 shows exemplary data collapses of $D$ [panel (a)] and $r$ [panel (b)] over the QP energies, $W / t=7$ and all system sizes $L$, where collapses have been performed for all the data in the region within the large black boxes in Figs. 2(d) and 2(e) with filled symbols marking the scaling result [52]. As a result of all collapses we find

$$
\beta / v=0.26(5), \quad 1 / v=0.91(4), \quad D_{c}=0.51(3),
$$

implying weak fractal behavior at the critical point. We note that these exponents are consistent with the Harris criterion [61-63] and those of the universality class for one- 
dimensional directed percolation $[64,65]$. While we get a good collapse for each individual disorder value (Fig. 3 and Supplemental Material [52]) deviations from a single line imply a weak dependency of $\tilde{D}_{W}$ and $\tilde{r}_{W}$ on $W$. Also, the decay of $r$ towards $r_{P}$ is always nearly exponential [black line in Fig. 2(c), and Supplemental Material]. From the collapsed data at the critical point we extract a thermal-like $r_{c}=$ $\left\langle\tilde{r}_{W}(0)\right\rangle_{W}=0.527(3) \approx r_{\mathrm{G}}$ consistent with the weak fractality of the critical QP states and $\left\langle\tilde{D}_{W}(0)\right\rangle_{W}=0.35(3)$. Here, $\langle\cdot\rangle_{W}$ is the average over $W$ inside the large boxes in Figs. 2(d) and 2(e).

Next, we determine two independent estimates of $\omega_{c}(W)$ for other $U / t$ at fixed $L=32$ and up to $N=5$, which is necessary to determine the low-energy ME at strong disorder. We take the crossing points of (i) $D$ data with the finite-size critical dimension $D_{c, L=32}=0.657$ (16) [Fig. 2(a), black arrow], as well as of (ii) exponential fits to $r$ data with the critical gap ratio $r_{c}$ [see black line in Figs. 3(b) and 2(a), black arrow] [66]. For $U / t \in\{3,20\}$, respectively, Fig. 2 shows the $D_{c, L=32}$ MEs [empty symbols, panels (b) and (d)] and binned $r$ data [six values per bin, panels (c) and (e)] close to the critical $r_{c}$. We note that $L=40$ for $N=5$ in panels (d) and (e) is necessary to resolve the low-energy ME as we discuss in the Supplemental Material. Excitingly, this procedure leads to consistent values for $\omega_{c}(W)$ for all considered values of $W$ and $U$. Interestingly, we find that for all data sets and sufficiently small $\omega$, the dependence of $\omega_{c}(W)$ on $W$ is consistent with the empirical ansatz

$$
\omega_{c}(W)=\omega_{0} \exp (-W / \Omega),
$$

except for small $U$ where the gap to the upper band already vanishes at small $W$. Corresponding exponential fits to the $N=3$ data, shown as thick black lines in Figs. 2(b)-2(e), work well in a large part of the spectrum, while additional data obtained by increasing $N$ and $L$ matches up perfectly for disorder values beyond the $N=3$ truncation limit [52]. Panel (b) of Fig. 1(b) summarizes these findings, showing the extension of delocalized QP states up to the ME $W_{c}(\omega)$ as a function of interaction at fixed energy $\omega=t$ with its greatest extension at $U / t \approx 15$, while the parameters of (11) are given in the inset of Fig. 1(b) depicting amplitudes $\omega_{0}$ and decay constants $\Omega$ as functions of $U / t$. We note that the perfect match of (11) for increased truncation and system sizes implies the absence of a thermal to fully QP localized phase, if extended to the thermodynamic limit [21-23].

Trapped system. We end our discussion with the analysis of the added effect of a harmonic trap as realized in [26] approximating the skewed Gaussian disorder used therein by an exact Gaussian with the full width at half maximum $\Delta=$ $2 \sqrt{2 \ln 2} W$. All other parameters of (1) are taken from the reference so $U=24.4 t$, the total particle number is 133 , and we set $L=32$ with $N_{r}=95$. In Fig. 4(a) we show the gap ratio of the QP spectrum related to a mean-field ground state with a central density of 1 surrounded by a condensate ring, contrary to the experiment which used a purely Mott-type initial state. The considered QP states localize at roughly the same energy scale as in the experiment, which we quantify by an exponential fit of $r$ for the least localized states at $\omega_{\gamma} / t \approx 0.1$ [see Fig. 4(b)] resulting in a finite-size transition at $\Delta_{c}^{(r)} / t=7.9(1.5)[67]$.
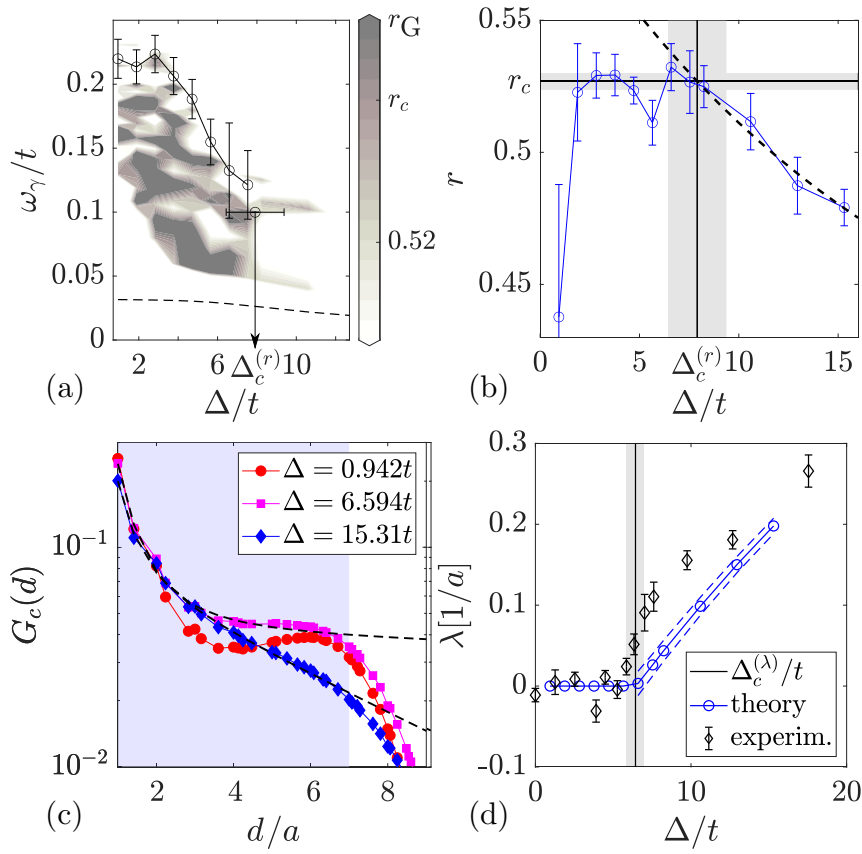

FIG. 4. Localization in a harmonically trapped lattice. (a) Binned (every six gap pairs) gap ratio contours of the QP spectra as function of $\Delta / t$. Circles mark the ME obtained from the crossing of an exponential fit to each $r\left(\omega_{\gamma}\right)$ with $r_{c}$ and the dashed line marks the lowest resolved QP excitation. (b) Circles are binned data for the $11 r$ values closest to $\omega_{\gamma} / t=0.1$ [between solid lines in (a)]. The crossing of the exponential fit (dashed line) with $r_{c}$ yields $\Delta_{c}^{(r)}$ [vertical arrow in (a)], both shown as black lines together with associated errors. (c) Exemplary fits of (12) to the numerical $G_{c}$ for various $\Delta$. (d) Inverse decay length $\lambda$ of (12) in comparison to experimental data [26]. The black solid line marks the theoretical prediction of $\Delta_{c}^{(\lambda)}$ together with one standard deviation.

To get further insight we consider the scaling of connected single particle correlations as given by $G_{c}\left(\ell, \ell^{\prime}\right) \equiv$ $\left\langle\left\langle\hat{b}_{\ell}^{\dagger} \hat{b}_{\ell^{\prime}}\right\rangle_{\mathrm{QP}}-\phi_{\ell}^{*} \phi_{\ell^{\prime}}\right\rangle_{d}$. Here $\langle\cdot\rangle_{\mathrm{QP}}$ is the QP ground state expectation value implicitly defined via $\beta_{\gamma}\left|\psi_{\mathrm{QP}}\right\rangle=0$ for all $\gamma$ [40-43], thus best fulfilling the original approximation of neglected QP interactions. We then consider the radial correlations of the four central sites averaged for each unique distance from the trap center [see Fig. 4(c)]. Due to the vicinity to a localization transition and the inhomogeneous nature of the system we expect an interplay of algebraic and exponential correlations which we summarize in the fit function $[52,68]$

$$
G_{c}(d)=a_{1} \exp (-\lambda d)+a_{2} d^{-b} .
$$

In Fig. 4(d) we show the various obtained inverse localization lengths $\lambda$ of these fits together with one standard deviation of the fitting error. Below a certain disorder strength we find no exponential contribution. A linear fit for all nonzero $\lambda$ yields the theoretical critical disorder strength $\Delta_{c}^{(\lambda)} / t=6.4(6) \mathrm{com}$ paring well to the experimental value $\Delta_{c} / t=5.3(2)$, which, to our knowledge, is the first theoretical prediction. The different slope compared to experiment likely stems from the slightly different nature of the considered observables. We note that the localization happens at a much smaller disorder 
strength than predicted for the unconfined system. This is most likely due to the trap enhanced variance of the local potential.

Conclusion. In conclusion, we have performed a detailed analysis of the two-dimensional BHM with Gaussian disorder at half filling by discussing gap ratios and fractal dimensions of generalized (beyond Bogoliubov) QP eigenstates. We find a strongly localized spectrum with at least one mobility edge separating a small fraction of delocalized noninteracting QP modes at low energies from high lying localized ones. For all converged results this critical line follows an exponential decay with disorder down to quasiparticle energies of order $0.1 t$. Finite-size scaling in the vicinity of these critical lines yields relevant critical exponents and parameters for a spectral transition characterized by a thermal-like gap ratio and weak multifractality. Furthermore, the MEs are strongly affected by the structure of QP bands in the clean system. Our method predicts a scaling of correlations almost identical to that observed in experiment [26] and the finite-size transition point without requiring any empirical fit parameter.
As we show in this work, the FOE is a very promising tool for the analysis of extended systems with strong correlations, which can also be used to clarify the interplay between MBL and the BG [69]. As the FOE can easily be extended to the time domain, it furthermore opens up an exciting direction of future research into disorder-driven dynamical effects.

Acknowledgments. A.G. would like to thank A. R. Abadal, C. Groß, L. Rademaker, and J. Schachenmayer for insightful discussions. Support by the Leopoldina Fellowship Programme of the German National Academy of Sciences Leopoldina Grant No. LPDS 2018-14, the ANR ERA-NET QuantERA-Projet RouTe (ANR-18-QUAN-000501), and the High Performance Computing Center of the University of Strasbourg, providing access to computing resources and scientific support, is gratefully acknowledged. Part of the computing resources were funded by the Equipex Equip@Meso project (Programme Investissements d'Avenir) and the CPER Alsacalcul/Big Data. G.P. is further supported by USIAS in Strasbourg and the Institut Universitaire de France (IUF).
[1] P. W. Anderson, Absence of diffusion in certain random lattices, Phys. Rev. 109, 1492 (1958).

[2] E. Abrahams, P. W. Anderson, D. C. Licciardello, and T. V. Ramakrishnan, Scaling Theory of Localization: Absence of Quantum Diffusion in Two Dimensions, Phys. Rev. Lett. 42, 673 (1979).

[3] L. Fleishman and P. W. Anderson, Interactions and the Anderson transition, Phys. Rev. B 21, 2366 (1980).

[4] G. Roati, C. D’Errico, L. Fallani, M. Fattori, C. Fort, M. Zaccanti, G. Modugno, M. Modugno, and M. Inguscio, Anderson localization of a non-interacting Bose-Einstein condensate, Nature (London) 453, 895 (2008).

[5] B. L. Altshuler, Y. Gefen, A. Kamenev, and L. S. Levitov, Quasiparticle Lifetime in a Finite System: A Nonperturbative Approach, Phys. Rev. Lett. 78, 2803 (1997).

[6] D. M. Basko, I. L. Aleiner, and B. L. Altshuler, Metal-insulator transition in a weakly interacting many-electron system with localized single-particle states, Ann. Phys. 321, 1126 (2006).

[7] V. Oganesyan and D. A. Huse, Localization of interacting fermions at high temperature, Phys. Rev. B 75, 155111 (2007).

[8] A. Pal and D. A. Huse, Many-body localization phase transition, Phys. Rev. B 82, 174411 (2010).

[9] M. Serbyn, Z. Papić, and D. A. Abanin, Local Conservation Laws and the Structure of the Many-Body Localized States, Phys. Rev. Lett. 111, 127201 (2013).

[10] D. A. Huse, R. Nandkishore, and V. Oganesyan, Phenomenology of fully many-body-localized systems, Phys. Rev. B 90, 174202 (2014).

[11] A. Chandran, I. H. Kim, G. Vidal, and D. A. Abanin, Constructing local integrals of motion in the many-body localized phase, Phys. Rev. B 91, 085425 (2015).

[12] V. Ros, M. Müller, and A. Scardicchio, Integrals of motion in the many-body localized phase, Nucl. Phys. B 891, 420 (2015).
[13] J. Z. Imbrie, V. Ros, and A. Scardicchio, Local integrals of motion in many-body localized systems, Ann. Phys. 529, 1600278 (2017).

[14] P. Sierant and J. Zakrzewski, Many-body localization of bosons in optical lattices, New J. Phys. 20, 043032 (2018).

[15] T. B. Wahl, A. Pal, and S. H. Simon, Signatures of the manybody localized regime in two dimensions, Nat. Phys. 15, 164 (2019).

[16] J. Z. Imbrie, On many-body localization for quantum spin chains, J. Stat. Phys. 163, 998 (2016).

[17] J. Z. Imbrie, Diagonalization and Many-Body Localization for a Disordered Quantum Spin Chain, Phys. Rev. Lett. 117, 027201 (2016).

[18] R. Nandkishore, Many-body localization and delocalization in the two-dimensional continuum, Phys. Rev. B 90, 184204 (2014).

[19] A. Kshetrimayum, M. Goihl, and J. Eisert, Time evolution of many-body localized systems in two spatial dimensions, arXiv:1910.11359 [Phys. Rev. B (to be published)].

[20] J. Šuntajs, J. Bonča, T. Prosen, and L. Vidmar, Quantum chaos challenges many-body localization, arXiv:1905.06345.

[21] W. De Roeck, F. Huveneers, M. Müller, and M. Schiulaz, Absence of many-body mobility edges, Phys. Rev. B 93, 014203 (2016).

[22] K. Agarwal, E. Altman, E. Demler, S. Gopalakrishnan, D. A. Huse, and M. Knap, Rare-region effects and dynamics near the many-body localization transition, Ann. Phys. 529, 1600326 (2017).

[23] W. De Roeck and F. Huveneers, Stability and instability towards delocalization in many-body localization systems, Phys. Rev. B 95, 155129 (2017).

[24] A. Lukin, M. Rispoli, R. Schittko, M. E. Tai, A. M. Kaufman, S. Choi, V. Khemani, J. Léonard, and M. Greiner, Probing entanglement in a many-body-localized system, Science (New York, N.Y.) 364, 256 (2019). 
[25] M. Rispoli, A. Lukin, R. Schittko, S. Kim, M. E. Tai, J. Léonard, and M. Greiner, Quantum critical behaviour at the many-body localization transition, Nature (London) 573, 385 (2019).

[26] J.-y. Choi, S. Hild, J. Zeiher, P. Schauß, A. Rubio-Abadal, T. Yefsah, V. Khemani, D. A. Huse, I. Bloch, and C. Gross, Exploring the many-body localization transition in two dimensions, Science (New York, N.Y.) 352, 1547 (2016).

[27] A. Rubio-Abadal, J.-y. Choi, J. Zeiher, S. Hollerith, J. Rui, I. Bloch, and C. Gross, Many-Body Delocalization in the Presence of a Quantum Bath, Phys. Rev. X 9, 041014 (2019).

[28] L. Fallani, J. E. Lye, V. Guarrera, C. Fort, and M. Inguscio, Ultracold Atoms in a Disordered Crystal of Light: Towards a Bose Glass, Phys. Rev. Lett. 98, 130404 (2007).

[29] C. Meldgin, U. Ray, P. Russ, D. Chen, D. M. Ceperley, and B. DeMarco, Probing the Bose glass-superfluid transition using quantum quenches of disorder, Nat. Phys. 12, 646 (2016).

[30] D. S. Fisher and M. P. A. Fisher, Onset of Superfluidity in Random Media, Phys. Rev. Lett. 61, 1847 (1988).

[31] M. P. A. Fisher, P. B. Weichman, G. Grinstein, and D. S. Fisher, Boson localization and the superfluid-insulator transition, Phys. Rev. B 40, 546 (1989).

[32] M. Makivić, N. Trivedi, and S. Ullah, Disordered Bosons: Critical Phenomena and Evidence for New Low Energy Excitations, Phys. Rev. Lett. 71, 2307 (1993).

[33] S. Zhang, N. Kawashima, J. Carlson, and J. E. Gubernatis, Quantum Simulations of the Superfluid-Insulator Transition for Two-Dimensional, Disordered, Hard-Core Bosons, Phys. Rev. Lett. 74, 1500 (1995).

[34] I. F. Herbut, Dual Superfluid-Bose-Glass Critical Point in Two Dimensions and the Universal Conductivity, Phys. Rev. Lett. 79, 3502 (1997).

[35] A. Priyadarshee, S. Chandrasekharan, J.-W. Lee, and H. U. Baranger, Quantum Phase Transitions of Hard-Core Bosons in Background Potentials, Phys. Rev. Lett. 97, 115703 (2006).

[36] Ş. G. Söyler, M. Kiselev, N. V. Prokof'ev, and B. V. Svistunov, Phase Diagram of the Commensurate Two-Dimensional Disordered Bose-Hubbard Model, Phys. Rev. Lett. 107, 185301 (2011).

[37] Juan Pablo Álvarez Zúñiga and N. Laflorencie, Bose-Glass Transition and Spin-Wave Localization for 2D Bosons in a Random Potential, Phys. Rev. Lett. 111, 160403 (2013).

[38] J. Saliba, P. Lugan, and V. Savona, Superfluid-insulator transition of two-dimensional disordered Bose gases, Phys. Rev. A 90, 031603(R) (2014).

[39] Juan Pablo Álvarez Zúñiga, D. J. Luitz, G. Lemarié, and N. Laflorencie, Critical Properties of the Superfluid-Bose-Glass Transition in Two Dimensions, Phys. Rev. Lett. 114, 155301 (2015).

[40] Ulf Bissbort, M. Buchhold, and W. Hofstetter, Quasi-particle theory for the Higgs amplitude mode, arXiv:1401.4466.

[41] A. Geißler, Ulf Bissbort, and W. Hofstetter, Quasiparticle spectra of supersolid lattice gases at near-resonant Rydberg dressing, Phys. Rev. A 98, 063635 (2018).

[42] Ulf Bissbort, Dynamical effects and disorder in ultracold bosonic matter, Ph.D. thesis, Johann Wolfgang GoetheUniversität, 2012.
[43] A. Geißler, Lattice-supersolids in bosonic quantum gases with Rydberg excitations, Ph.D. thesis, Johann Wolfgang GoetheUniversität, 2018.

[44] A crucial difference compared to the commonly considered box disorder is the presence of rare extreme peaks or wells in the potential.

[45] We refer the interested reader to our follow-up work [69] for a detailed discussion of the FOE method in the context of disordered systems.

[46] We note that the MF self-consistency condition guarantees the absence of first order terms.

[47] S. D. Huber, E. Altman, H. P. Büchler, and G. Blatter, Dynamical properties of ultracold bosons in an optical lattice, Phys. Rev. B 75, 085106 (2007).

[48] S. D. Huber, B. Theiler, E. Altman, and G. Blatter, Amplitude Mode in the Quantum Phase Model, Phys. Rev. Lett. 100, 050404 (2008).

[49] Ulf Bissbort, S. Götze, Y. Li, J. Heinze, J. S. Krauser, M. Weinberg, C. Becker, K. Sengstock, and W. Hofstetter, Detecting the Amplitude Mode of Strongly Interacting Lattice Bosons by Bragg Scattering, Phys. Rev. Lett. 106, 205303 (2011).

[50] M. Endres, T. Fukuhara, D. Pekker, M. Cheneau, P. Schauß, C. Gross, E. Demler, S. Kuhr, and I. Bloch, The 'Higgs' amplitude mode at the two-dimensional superfluid/Mott insulator transition, Nature (London) 487, 454 (2012).

[51] N. N. Bogolyubov, On the theory of superfluidity, J. Phys. (USSR) 11, 23 (1947) [Izv. Akad. Nauk Ser. Fiz. 11, 77 (1947)].

[52] See Supplemental Material at http://link.aps.org/supplemental/ 10.1103/PhysRevResearch.2.042037 for a discussion of details of the finite-size scaling analysis for the excitations and the ground state, a comparison of scenarios for the decay of correlations in a trapped system, and a discussion of the entanglement captured by the FOE method.

[53] I. Frérot and T. Roscilde, Area law and its violation: A microscopic inspection into the structure of entanglement and fluctuations, Phys. Rev. B 92, 115129 (2015).

[54] I. Frérot and T. Roscilde, Entanglement Entropy Across the Superfluid-Insulator Transition: A Signature of Bosonic Criticality, Phys. Rev. Lett. 116, 190401 (2016).

[55] C. Gaul, P. Lugan, and C. A. Müller, Anderson localization of Bogoliubov excitations on quasi-1D strips, Ann. Phys. 527, 531 (2015).

[56] Y. Y. Atas, E. Bogomolny, O. Giraud, and G. Roux, Distribution of the Ratio of Consecutive Level Spacings in Random Matrix Ensembles, Phys. Rev. Lett. 110, 084101 (2013).

[57] H. G. E. Hentschel and I. Procaccia, The infinite number of generalized dimensions of fractals and strange attractors, Phys. D (Amsterdam, Neth.) 8, 435 (1983).

[58] N. Macé, F. Alet, and N. Laflorencie, Multifractal Scalings Across the Many-Body Localization Transition, Phys. Rev. Lett. 123, 180601 (2019).

[59] J. Lindinger, A. Buchleitner, and A. Rodríguez, Many-Body Multifractality throughout Bosonic Superfluid and Mott Insulator Phases, Phys. Rev. Lett. 122, 106603 (2019).

[60] From here on we omit the indices of the observables unless they are necessary.

[61] A. B. Harris, Effect of random defects on the critical behaviour of Ising models, J. Phys. C 7, 1671 (1974). 
[62] J. T. Chayes, L. Chayes, D. S. Fisher, and T. Spencer, FiniteSize Scaling and Correlation Lengths for Disordered Systems, Phys. Rev. Lett. 57, 2999 (1986).

[63] T. Vojta and J. A. Hoyos, Criticality and Quenched Disorder: Harris Criterion Versus Rare Regions, Phys. Rev. Lett. 112, 075702 (2014).

[64] I. Jensen, Low-density series expansions for directed percolation: I. A new efficient algorithm with applications to the square lattice, J. Phys. A: Math. Gen. 32, 5233 (1999).

[65] J. Wang, Z. Zhou, Q. Liu, T. M. Garoni, and Y. Deng, Highprecision Monte Carlo study of directed percolation in $(d+1)$ dimensions, Phys. Rev. E 88, 042102 (2013).
[66] Most of the time the exponential gap ratio fits slightly overestimate the critical energy, but always well within the errorbars.

[67] Extremely small values of $r$ at small $\Delta$ and $\omega$ in Fig. 2(a) result from nearly degenerate low-energy pairs due to an approximate discrete rotational symmetry.

[68] K. S. Tikhonov and A. D. Mirlin, Statistics of eigenstates near the localization transition on random regular graphs, Phys. Rev. B 99, 024202 (2019).

[69] A. Geißler, Finite-size scaling analysis of localization transitions in the disordered two-dimensional Bose-Hubbard model within the fluctuation operator expansion method, arXiv:2011.10104 